\title{
Understanding User Preferences of Digital Privacy Nudges - A Best-Worst Scaling Approach
}

\author{
Sofia Schöbel \\ University of Kassel \\ $\underline{\text { sofia.schoebel@uni-kassel.de }}$ \\ Felix Hupfeld \\ University of Kassel \\ felix.hupfeld@wi-kassel.de
}

\author{
Torben Jan Barev \\ University of Kassel \\ torben.barev@uni-kassel.de
}

\author{
Andreas Janson \\ University of Kassel \\ andreas.janson@uni-kassel.de
}

Jan Marco Leimeister

University of Kassel \&

University of St. Gallen

janmarco.leimeister@unisg.ch

\begin{abstract}
Digital nudging in privacy has become more important to protect users of information systems while working with privacy-related data. Nudging is about altering a user's behavior without forbidding any options. Several approaches exist to "nudge" users to change their behavior. Regarding the usage of digital privacy nudges, research still has to understand the meaning and relevance of individual nudges better. Therefore, this paper compares the preferences of users for different digital nudges. To achieve this goal, it presents the results of a so-called best-worst scaling. This study contributes to theory by providing a better understanding of user preferences regarding design variations of digital nudges. We support practitioners by giving implications on how to design digital nudges in terms of user preferences.
\end{abstract}

\section{Introduction}

Due to the increasing relevance of digitalization in private and work lives, nowadays, more decisions are made online by visiting websites or using mobile apps [1]. Although digitalization offers innovation potential for business and makes the lives of individuals easier, there are also tremendous risks [2]. Individuals share information with others, not only in their daily lives but also within their company. Such privacy risks especially relate to issues like individuals leaving data traces in every working step on internet platforms such as Wikis or on external work tools such as Slack while oftentimes not being aware of their generated data [3].

To handle privacy-related information better, solutions for information systems (IS) are necessary that mitigate privacy risks and foster information privacy.
One solution is the usage of so-called "digital nudges", which are different goal-oriented elements that are used in blended as well as digital environments with the intention to influence individuals judgements, their choices or behavior [4]. Referring to the issue of privacy in digital environments, nudges should help users to make better privacy decisions in their personal and professional lives.

However, some challenges exist about the usage of digital nudges and the preferences of users. In terms of digital nudge designs, most nudging concepts are designed for the average user without adapting them to a specific group of users or a specific context (such as the context of privacy) [5].There is some evidence that users have preferences for nudges in terms of their characteristics, and designing nudges by considering user preferences becomes increasingly important to improve their effectiveness $[6,7]$.

To understand user preferences concerning different digital privacy nudges better, it is important to identify existing variations of designs in a first step, which can be compared by users in terms of their preferences in a second step. Consequently, the goal of our paper is to analyze which digital privacy nudges users prefer in general to get a better understanding of how to design privacy nudges in digital environments. Therefore, our paper focusses on the following research question (RQ):

\section{$R Q:$ Which digital privacy nudges do users prefer?}

To answer our RQ, we present the results of a socalled best-worst scaling (BWS) [8] approach. BWS helps to analyze user preferences by asking users which object out of a list of three or four they prefer and which not [8]. A BWS delivers a ranking of objects indicating which objects users prefer the most (first rank) and which the least (last rank). With such a ranking, we can better analyze which digital nudges 
in the context of privacy have to be analyzed in more detail to make them more preferred by users.

We provide theoretical implications of each nudge and their relation to user preferences. In addition, we describe the characteristics of each nudge in detail. We offer recommendations about how to use a BWS to learn more about the relevance of preferences in digital environments. We provide practical implications for system developers about how to design more meaningful digital privacy nudges.

The remainder of the paper is structured as follows. After motivating our research idea, we will describe digital nudging and present related work on nudging and privacy. Next, we will present the method we used and will continue with the description of the results. Finally, we will discuss the results and will outline the contributions as well as the limitations of our paper.

\section{Theoretical Background}

This paper focusses on digital nudging and on privacy-related issues. In the following, we discuss the terms digital nudges and the role of privacy. We will refer to the privacy paradox and calculus to better understand the role of digital nudges in privacy.

In the second part of this section, we will present the dual-process theory which is important to understand how users react towards different digital nudges.

\subsection{Digital Nudges and the Context of Privacy}

Nudging has its origins in offline settings endorsed by behavioral economics. A nudge is defined as "any aspect of the choice architecture that alters people's behavior in a predictable way without forbidding any options or significantly changing their economic incentives" [9, p. 6].

In IS research, nudging has become more and more relevant and has led to the concept of digital nudging $[4,10]$. Today's decisions are made online and digital nudging can support individuals in guiding them in a certain direction [11]. At the same time, digital environments offer significantly different options for nudging compared to offline environments [4].

Digital nudging is present in many different areas such as privacy, crowdfunding, or e-commerce [4]. Originally, digital nudging has been defined as "the use of user-interface design elements to guide people's behavior in digital choice environments" [12, p. 433]. These choice environments especially relate to all user interfaces where individuals make decisions.

Another definition of digital nudging can be found in Meske and Potthoff's [13] work. They define digital nudges as "a subtle form of using design, information, and interaction elements to guide user behavior in digital environments, without restricting the individual's freedom of choice" [13, p. 2589].

However, both definitions are not precise about the elements of digital nudging which cannot be easily transferred from offline nudges [4]. Lembcke et al. [4] consider the role of nudges in their work and describe a digital nudge as "any intended and goal-oriented intervention element (e.g. design, information or interaction elements) in digital or blended environments attempting to influence people's judgment, choice or behavior" $[4$, p. 10].

Kissmer et al. [14], and Kroll [11] compare the concept of nudging with persuasion which is described as a form of communication between individuals with the aim to influence autonomous judgements and actions of individuals [15]. A persuasive technology on the other hand changes the attitudes and behavior of individuals [15]. Some similarities can be found when comparing nudging and persuasive technologies like the fact that both indent to change an individual's behavior or that they do not forbid a user any options [14]. For this work, we will refer to the definition of Lembcke et al. [4], because they consider the role and meaning of nudging "elements" which are an important part of our work. Whereas persuasive technologies influence decision making, digital nudging refers to biases and heuristics that try to lead users to beneficial decisions and at the same time to preserve the users freedom of choice through modifications of the digital choice environment [16]

Regarding the usage of digital nudges, one important stream of literature and research has focused on privacy-related topics [3]. Privacy has become more relevant not only in IS research. In social media, individuals oftentimes share content with other users that is often inconsistent with their own intentions and they are oftentimes not able to manage their own privacy settings [17]. Individuals disclosure personal information without protecting behaviors which can be described as privacy paradox [17, 3].

In addition, the so-called privacy calculus is of relevance by which individuals rationally weigh potential benefits and risks before making a decision [18]. In IS, users might exchange personal data in exchange for time and money, self-enhancements, or pleasure [19].

Having the privacy paradox and calculus in mind, several recommendations can support users in protecting their privacy data [17]. One solution can be the use of digital nudges [3]. With digital nudges, users might be able to better (and faster) protect their data. In summary, offline nudges cannot be used in and applied to the digital environment [4]. It remains unclear which and how offline nudges can be transferred to online settings [10]. 
In addition, less is known about how to design effective digital privacy nudges to change the users behavior [20]. To make better judgements about how users react towards different nudges it is necessary to understand which digital nudges exist and in addition to understand how users react towards different digital nudges. With an effective nudging concept and a better understanding about each nudge, aspects such as the privacy paradox or calculus could be better handled.

\subsection{Underlying Mechanisms of Digital Nudges}

Particularly in the context of decisions relating to privacy, human decision-making is often imperfect and decisions are made that often do not correspond to the desired objectives. Studies have shown that especially users of digital systems often act irrationally due to cognitive, emotional and social factors [3, 9].

Potential explanations can be found in the dualprocess theory, which states that users use two systems of thought [21]. Two systems are therefore necessary to better evaluate the abundance of information in today's (digital) world and to make targeted decisions. System 1 represents our intuitions or our unconscious autopilot. System 2, on the other hand, expresses itself through our conscious planning and control. However, system 2 requires significantly more mental effort and time. Both systems are active at the same time and usually work together smoothly [21].

In everyday life, however, users rarely have enough time and information to fully evaluate all alternatives. Instead of exercising a systematic decisionmaking process, users tend to resort to so-called heuristics (mental abbreviations) [22]. Heuristics are informal rules of thumb that reduce the complexity of decision-making and thus represent abbreviations in decision-making. Although heuristics are an efficient way to solve recurring problems, they can lead to systematic errors such as biases in information evaluation [23].

For example, personal data is often disclosed carelessly because the risk of unwanted monitoring is less present mentally (availability heuristics). These false conclusions do not mean that the behavior of users is unpredictable and irrational. Rather, it is a systematic and thus predictable deviation from rational behavior.

This is where digital privacy nudges come into play. Privacy nudges can influence both systems of thought by exploiting heuristics or counteracting them in order to guide users to their informational self-determination [12]. Interestingly, the perceived aspect in the choice environment guiding users' behavior, for instance, a colored element or given information, can be processed differently by users [24]. Some stimuli may be perceived as pleasant, while others may be perceived as unpleasant. The initial stimuli may therefore be crucial for the nudge effectiveness and are worth further investigation. It is worth exploring how users perceive specific nudges [6].

\section{Related Work about Digital Privacy Nudges}

To analyze which digital privacy nudges users prefer, we first have to get a better understanding of existing nudges and their categorization. In doing so, we conducted a systematic literature search [25] to identify which digital nudges and classifications of nudges exist. The following databases were included: ACM Digital Library, AIS Electronic Library (AISeL), EBSCOHost, Emerald Insight, Institute of Electrical and Electronic Engineers (IEEE), SSRN. To cover a broad set of publications, the keyword "privacy nudge" was used. The number of identified and reduced papers is shown in Figure 1.

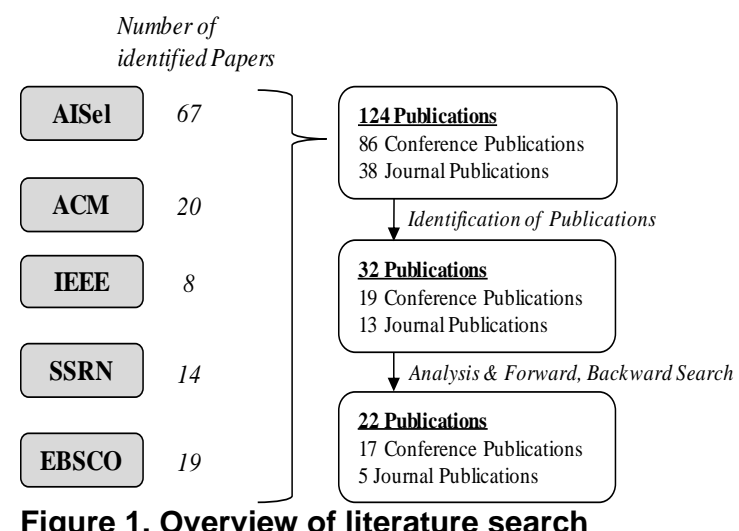

The initial number of 124 papers was reduced by reading the papers' title, their abstracts, and keywords. We excluded papers that did not focus on nudging. In addition, we excluded studies that were not relevant for the context of privacy and also excluded studies that were not written in English or German. Finally, we excluded duplicates.

In a second step, the remaining papers were read carefully to identify those papers that focused on privacy nudges and their description and design. We included studies identified by cross referencing. In the end, 22 papers remained for the identification of nudge designs in digital environments. Each of the 22 papers we analyzed was read carefully to identify which digital nudges each study used. Such an understanding is necessary to conduct a BWS. More precisely, we used the results of our literature review to derive a typology of digital privacy nudges which is presented in Table 1. We used the results of prior research studies about digital nudges and nudges in general to identify different groups of nudges. Here, we could identify seven 
different groups of nudges that are relevant for our study: Defaults, presentation, information, feedback, error, social influence. In addition to exemplary literature sources, the typology shows the special biases, heuristics and principles that privacy nudges exploit or mitigate.

Table 1. Digital privacy nudges

\begin{tabular}{|l|l|}
\hline $\begin{array}{l}\text { Privacy } \\
\text { Nudges }\end{array}$ & Explanation \\
\hline Default & $\begin{array}{l}\text { Preselected options in a system that are set as de- } \\
\text { faults, predetermining the extent to which private } \\
\text { data is shared [3]. }\end{array}$ \\
\hline $\begin{array}{l}\text { Presenta- } \\
\text { tion \& }\end{array}$ & $\begin{array}{l}\text { Data are presented by color cues to convey the } \\
\text { expected privacy risk in an online environment } \\
\text { [26]. }\end{array}$ \\
\hline $\begin{array}{l}\text { Infor- } \\
\text { mation }\end{array}$ & $\begin{array}{l}\text { Providing information in online privacy-related } \\
\text { decision-making situations to enable a realistic } \\
\text { perspective on privacy infringement risks [27]. }\end{array}$ \\
\hline Feedback & $\begin{array}{l}\text { Feedback is provided alongside and after the pri- } \\
\text { vacy-sensitive processes to inform the user about } \\
\text { the consequences of their actions [3]. }\end{array}$ \\
\hline $\begin{array}{l}\text { Error Re- } \\
\text { siliency }\end{array}$ & $\begin{array}{l}\text { Expecting users to make privacy protection er- } \\
\text { rors and allowing them to recover from them } \\
\text { [27]. }\end{array}$ \\
\hline $\begin{array}{l}\text { Social In- } \\
\text { fluence }\end{array}$ & $\begin{array}{l}\text { Visualization of how other users have behaved or } \\
\text { not behaved in terms of specific decisions by pic- } \\
\text { tures or textual elements [28]. }\end{array}$ \\
\hline $\begin{array}{l}\text { Progress } \\
\text { Bar }\end{array}$ & $\begin{array}{l}\text { A bar graph visualizes the progress of a certain } \\
\text { activity in a system and is intended to motivate } \\
\text { the completion of this activity [29]. }\end{array}$ \\
\hline
\end{tabular}

The design element of default privacy nudges describes the preselection of alternatives. As users often do not adapt online privacy settings to their needs, the default option (status-quo) remains overly preferred and mostly unchanged (status-quo bias) [3, 9]. In addition, the default option is used as a reference point for weighing decision options. This "anchor" is perceived unconsciously by users. Each decision option is now weighed against this alternative and the decision behavior is influenced in this direction [23]. Research about presentation and framing nudges exist when two identical alternatives influence the user's decision-making behavior differently due to their different presentation [26]. For example, colored fonts draw attention to selected elements in order to emphasize certain decision alternatives. By presenting nudges in combination with different colors like red or green elements, they can be framed in different ways to attract the attention of users.

Regarding information privacy nudges, the probability of privacy violations is often incomprehensible for users and underestimated [3, 23]. In digital nudging "information aims at mitigating negative effects of asymmetric and at overcoming availability and overconfidence biases that may lead to suboptimal decisions" [3, p. 13]. In order to counteract these negative effects, it is suggested that nudges inform information system users about the risks and consequences of the actions. Based on this information nudge design, the individual can make a well-founded decision about their own privacy [3].

A further privacy nudge design element is the provision of feedback, which indicates the previous usage behavior of a person. This nudge mechanism creates awareness of individual's previous and current decisions and their consequences [3]. Research that analyses the feedback privacy nudge covers mainly framing effects, hyperbolic discounting and in large parts the state of incomplete information. Error resiliency privacy nudges can assist users, as decisions on privacy often favor risky and ill thought-through decisions without taking possible long-term consequences into account. This is based on so-called hyperbolic discounting, in which the immediate benefit is overestimated, and costs incurred later are underestimated by users [3]. To counteract this, a time delay can be used as a privacy nudge [27]. In this way, the individual should be persuaded to act less impulsively and to rethink the message and possible negative consequences [3]. To understand this privacy nudge characteristic better, much of the current research is devoted to analyzing hyperbolic discounting, loss aversion effects and the state of incomplete information.

The effect of social influence privacy nudges is based on the principle of social norms. The individual derives to what extent it is appropriate to share personal information from the behavior of his fellow users [30, 31]. The majority's decision influences the perception and the behavior of users in a way [28] that others get the feeling of trying to imitate the behavior of the majority [31]. The more people have the same opinion on a particular topic, the more likely it is to elicit the same opinion in others [27] because behavior of like-minded people leads to individual behavior [32]. Besides cognitive effects, research analyses the influence of personality traits that determine the effectiveness of this nudge and suggests that differences in personality traits such as impulsivity, sociability and risk-taking are influencing the effectiveness of social influences.

Finally, progress bars were identified as privacy nudge mechanisms. Normally, a progress bar is used to document the users progress of completing an online profile he or she has to edit [11]. Regarding privacy issues, progress bars are for example used to highlight the degree of how much privacy-related information is shared or to visualize password strength [29]. The results of the literature review are used for BWS, which is described in the following.

\section{Methodology}

To analyze which digital nudges users prefer, we describe the BWS method in the next section method. 


\subsection{Best-Worst Scaling}

To measure user preferences, several methods exist. The aim of our research study is to identify which kind of digital privacy nudges users prefer. We decided to use a MaxDiff scaling to measure user preferences because we wanted an individual rating of each nudge. One approach that refers to MaxDiff scaling is the so-called "Best-Worst Scaling" (BWS). BWS was developed by Louviere and Woodworth [33], and it is an extension of the MaxDiff scaling that was originally developed by Thurstone [34]. BWS describes a cognitive process in which users repeatedly choose two objects that they feel exhibit the largest perceptual difference on a described continuum of interests in varying sets of three or more objects in a survey [35]. In comparison to other preference-based measurement methods, BWS has several advantages. First, it provides a high level of ranking information because each decision for a pair of attributes provides implications for the attribute that was not chosen [36]. Furthermore, it is scale-free, which prevents response styles and therefore does not affect the mean value and the variance [37]. Finally, other response biases can be avoided by using BWS [37]. Overall, comparisons with other rating methods show that BWS provides better results regarding the discrimination between different attributes [37, 38]. We therefore use a case 1 BWS and let participants choose between different objects, that is, which object they would prefer and which one they do not like and evaluated the MaxDiff model.

\subsection{Operationalization of Digital Privacy Nudges}

A BWS is used to compare the preferences of users for different objects [35]. In this paper, we want to compare which digital privacy nudges users prefer and which ones they do not. Consequently, it is necessary to operationalize nudges. Our section about related work presents seven different kinds of privacy nudges (see Table 1). For the operationalization, it is important to decide on the context in which the different privacy nudges are presented to a user. We decided to use Slack, which is a well-known web-based messaging service that is used in companies to communicate with coworkers. An operationalization for each privacy nudge and its visual representation is presented in Figure 2. Besides presentation and framing and error resiliency, all nudges could be designed based on the recommendations that are presented in Table 1. For the presentation \& framing nudge, several possibilities exist to design a privacy nudge. We decided to use colors that are well established digital nudges in the group of presentation \& framing nudges. Colors can be used to create different feelings [39]. Red elements create awareness of the fact that a user might publish privacyrelated data. Green elements signalize that no privacyrelated data is going to be published. For error resiliency, we used a counter that delays publishing material in Slack. The nudges were presented with pictures and additional descriptions.

\begin{tabular}{|c|c|}
\hline $\begin{array}{l}\text { Operationalization of } \\
\text { Nudges }\end{array}$ & Visual Representation of Nudges \\
\hline $\begin{array}{l}\text { Default: a button is used that } \\
\text { sets all options of a channel as } \\
\text { defaults. }\end{array}$ & $\begin{array}{l}\text { Privat } \\
\text { By default, these channels are private } \\
\text { Closed channelscan only be used with an } \\
\text { invitation an are not visible in the channel list }\end{array}$ \\
\hline $\begin{array}{l}\text { Presentation } \& \text { Framing- Red } \\
\text { Element: a red-colored button } \\
\text { indicates that a user is going to } \\
\text { create a public channel that } \\
\text { can be seen by all coworkers. }\end{array}$ & $\begin{array}{l}\text { Public } \\
\text { All workspace members can join }\end{array}$ \\
\hline $\begin{array}{l}\text { Presentation \& Framing - } \\
\text { Green Element: a green- } \\
\text { colored button indicates that } \\
\text { a user is going to create a } \\
\text { priva te channel. Only } \\
\text { coworkers that a re invited can } \\
\text { join. }\end{array}$ & $\begin{array}{l}\text { Privat } \\
\text { Closed channels can only be used with an } \\
\text { invitation and are not visible in the channel list. }\end{array}$ \\
\hline $\begin{array}{l}\text { Information: Before } \\
\text { publishing files in a channela } \\
\text { user is informed a bout which } \\
\text { coworkers can see a file that is } \\
\text { going to be published with }\end{array}$ & 19. An Anna, Andreas, Nicole, and 26others \\
\hline $\begin{array}{l}\text { that can see the message and } \\
\text { an additional text is provided. }\end{array}$ & \\
\hline $\begin{array}{l}\text { Feedback: a speech bubble } \\
\text { provides feedback to a user } \\
\text { a bout which privacy-related } \\
\text { information can be seen by } \\
\text { other coworkers. }\end{array}$ & $\begin{array}{l}\text { You are sharing } 64 \% \text { of your messages in a } \\
\text { public channel. } \\
\text { In average } 38 \text { people can see your messages. } \\
80 \% \text { of your personal informations can be seen } \\
\text { in your profile. }\end{array}$ \\
\hline $\begin{array}{l}\text { Time Delay: a counter is used } \\
\text { that delays publishing a } \\
\text { document in a channel. }\end{array}$ & $\begin{array}{l}\text { This message is going to be published in } 5 \\
\text { seconds }\end{array}$ \\
\hline $\begin{array}{l}\text { Social Nudge: a user is } \\
\text { informed a bout how many of } \\
\text { his coworkers have published } \\
\text { their phone number. }\end{array}$ & $\begin{array}{l}75 \% \text { of your colleagues do not share their } \\
\text { phone number with others. }\end{array}$ \\
\hline $\begin{array}{l}\text { Progress Bar: a progress bar } \\
\text { indicates the percentage of } \\
\text { privacy-related information } \\
\text { that is published. The red part } \\
\text { of the progress bar presents } \\
\text { priva cy-related data, the } \\
\text { green part protected data. }\end{array}$ & You have published $80 \%$ of your private informations \\
\hline
\end{tabular}

Figure 2. Operationalization and visualization of digital privacy nudges

\subsection{Data Collection for BWS}

To collect the data for our BWS, we used an online survey. The survey consisted of two parts. The first part was focused on the BWS task. In as second step, we asked for demographics and included questions about the participants' experience with Slack.

To construct the BWS task, choice sets need to be derived which represent a varying set of four different privacy nudges. An example of a choice set and the nudge presentation is presented in Figure 3. 


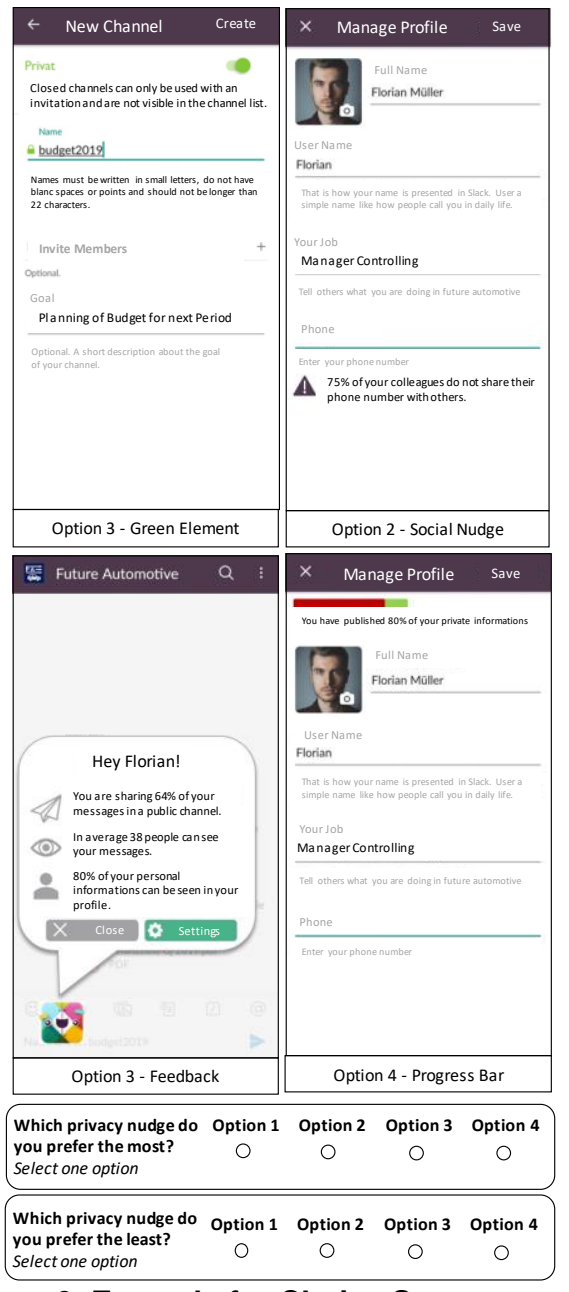

Figure 3. Example for Choice Set

The choice sets were constructed due to the use of BWS for the evaluation of user preferences. In general, $2^{\mathrm{k}}$ choice sets are necessary to obtain valid results [40]. In this case, $\mathrm{k}$ stands for the number of attributes in the analysis. To reduce the number of choice sets, most studies use a balanced incomplete block design (BIBD), which offers a smaller amount of choice sets to nonetheless receive valid results [33, 41, 42]. A BIBD is a type of design in which each choice option (i.e., privacy nudges) appears and co-appears equally often with each other choice option [37, 8]. To constitute the choice sets, we followed the four guidelines by Orme [43]. First, he recommends to show four or five attributes per choice. In our BWS we showed four privacy nudges per choice set. Second, each attribute should be shown three or more times to each respondent. For our analysis, we showed each privacy nudge seven times to each respondent. Third, each item should be shown just once in a choice set. We presented four different items (privacy nudges) in one choice set. Finally, for 10 or less items, there should only be a maximum amount of around 15 choice sets; we used 8 items (nudges) with 14 choice sets. To avoid order effects, we changed the position of the privacy nudges [40].

To collect our data, we transferred our choice sets to a web survey tool and pre-tested it. In our web survey, we first presented a figure and description of each digital nudge to the user. Next, users had to read the text for each nudge. In a second step, we started with the BWS task. As described above, a user had to rate each choice set. A choice set presents four digital nudges in Slack. We placed each nudge in the interface of Slack (see Figure 3) and asked the users to decide which of these four nudges he prefers the most and which one the least. We included an instruction manipulation check asking users to "select option 2" to guarantee that they read the instructions and were not randomly selecting options [44]. After the user had answered each task, we asked them about demographic data and their privacy concerns.

The pre-test was used to identify linguistic errors and to evaluate if the questions were understandable and free of mistakes. Apart from the correction of some grammar mistakes, our pre-testers indicated that the survey is understandable and that the privacy nudges can be identified in each screenshot.

After pre-testing the survey, we started our data collection. Participants were recruited via social media, mail or personally. In total, we were able to obtain 177 completed and usable surveys for our analysis. Data were collected in Germany over two month. Overall, 108 (61.02\%) participants were female and 69 (38.98\%) male. The youngest participant was 15 years old and the oldest 65 years old. The participants' average age was 26,85 years. Among others, most of our participants (85 participants, $48.02 \%$ ) had a university degree and $67(37.85 \%)$ of them had a general qualification for university entrance (20 participants $(11.30 \%)$ had a certificate for secondary education, three $(1.69 \%)$ of our participants were pupils, two participants $(1.13 \%)$ had an advanced technical certificate). In addition, most participants $(120,67.80 \%)$ were students, followed by $32(18.08 \%)$ participants with a part-time employment and $31(17.51 \%)$ with full-time employment (25 participants (14.21\%) had a mini-job, eight $(4.52 \%)$ were not working regularly, four $(2.26 \%)$ were in training, three participants $(1.69 \%)$ were pupils, two participants $(1.13 \%)$ were retired and one participant (1.13\%) was unemployed).

\section{Results of BWS}

All nudges that are demonstrated in our typology were used for the BWS analysis. Besides conducting a so-called counting analysis to calculate the results of our BWS, we calculated a logistic regression. The 
conditional logistic regression can be used to verify the ranking of the BWS to guarantee that the position calculated in the counting analysis is correct $[43,36]$ (see Table 2).

Table 2: Results BWS

\begin{tabular}{|c|c|c|c|c|c|c|c|}
\hline \multirow{2}{*}{$\begin{array}{l}\text { Ele- } \\
\text { ment }\end{array}$} & \multicolumn{4}{|c|}{ Counting Analysis } & \multicolumn{2}{|c|}{ Regression } & \multirow[t]{2}{*}{ Rank } \\
\hline & B & $\mathbf{W}$ & M & SD & Coe. & SD & \\
\hline Default & 570 & 123 & 0.36 & 0.47 & 1.08 & 0.057 & 1 \\
\hline Red & 392 & 204 & 0.15 & 0.49 & 0.69 & 0.055 & 2 \\
\hline Green & 326 & 202 & 0.10 & 0.46 & 0.59 & 0.055 & 3 \\
\hline $\begin{array}{l}\text { Feed- } \\
\text { back }\end{array}$ & 320 & 343 & $\overline{-}-\overline{0}$ & 0.57 & 0.37 & 0.054 & 4 \\
\hline $\begin{array}{l}\text { Infor- } \\
\text { mation }\end{array}$ & 289 & 351 & $\overline{0}-\overline{0}$ & 0.52 & 0.31 & 0.055 & 5 \\
\hline $\begin{array}{l}\text { Social } \\
\text { Nudge }\end{array}$ & 156 & 348 & $\overline{-}-15$ & 0.41 & 0.12 & 0.055 & 6 \\
\hline $\begin{array}{l}\text { Time } \\
\text { Delay }\end{array}$ & 230 & 436 & $\overline{0}-16$ & 0.55 & 0.10 & 0.055 & 7 \\
\hline $\begin{array}{l}\text { Pro- } \\
\text { gress } \\
\text { Bar }\end{array}$ & 195 & 471 & $\begin{array}{l}- \\
0.22\end{array}$ & 0.52 & - & - & 8 \\
\hline $\begin{array}{l}\mathrm{B}=\mathrm{Best}, \\
\text { cial Nu} \\
\mathrm{p}<0.001\end{array}$ & & & Iean, & $\mathrm{CSt}$ & ard & sion, B & $\begin{array}{l}\text { les So- } \\
\text { cant at }\end{array}$ \\
\hline
\end{tabular}

The results show that the most preferred privacy nudges are defaults (first ranking position), red elements (second ranking position), and green elements (third ranking position). All other privacy nudges had a lower ranking position. These elements were picked more often as worst nudges instead of best nudges. Feedback, for example, was picked 320 times as the best nudge and 343 times as the worst nudge. On the seventh ranking position, time delay is picked as nudge that is not much preferred by Slack users. On the last position, a progress bar was selected 471 times as the worst nudge and only 195 times as the most preferred privacy nudge.

\section{Discussion and Contributions}

In the following, we discuss the results and propose theoretical as well as practical implications.

Default nudges are in first place in the BWS, revealing the potential for designers to exploit the status quo bias. Here, the collected data suggests that privacy protection by default nudges sparks an individual's most positive initial reaction in digital work environments. In addition to defaults, only the two nudges in the form of presentation \& framing (red and green) were selected more often as preferred than not preferred elements. Specifically, the time delay, social or progress bar nudges are rated lower, as they may be perceived as disturbing and distracting in the individual's workflow. This may be the case as processing these nudges tends to require more cognitive effort [45]. With a progress bar, a user might not be able to identify the most critical privacy-related information whereby default options can automatically guarantee better privacy secured data in online environments. A red signal may be cognitively closely linked to the action of "stop" and does not need much interpretation by the individual. The green element may need more cognitive effort, as an interpretation of it is needed, and loss aversion bias does not accelerate the decisionmaking process. Respectively, these nudges tend to tackle system 2 thinking. System 2 expresses itself through our conscious planning and control. According to that, our data suggests that in privacy-related decision-making users perceive nudges as more positive when the nudges requires less cognitive work. Interestingly, the red element nudge is perceived more positively than the green element nudge. This might be due to the same effect. This phenomenon would therefore support our conclusion that users in privacy-related decisions perceive system 1 nudges as more pleasant than system 2 nudges.

The results also highlight that some nudges might be more intuitively to support the user's workflow than others. Time delay was ranked on the seventh position. In this respect, the time delay in particular may be perceived as disturbing or annoying, which in practice could also lead to ignorance and refusal of the nudge.

Nudges such as information and feedback might also be time consuming. Each time a user receives feedback or information he has to read the instructions such as " $65 \%$ of your private data are visual for all other users" and at the same time they have to figure out which data are part of these $65 \%$. In contrast, the results of the BWS show that presentation \& framing elements in form of colors are particularly suitable for guiding users intuitively and uncomplicatedly in the direction of certain decision alternatives without interrupting their work. The simple but strong effect of presentation \& framing elements and colors is confirmed in various contexts [46, 26]. User interface designers of digital work environments should therefore pay high attention to the design in which privacy nudges are supposed to reach the individual [47].

The results of this study indicate how important it is to consider users and their preferences when designing nudge concepts. We now know which nudges they prefer and which not. However, user preferences in nudging are just of relevance for users that are interested in protecting their privacy data in online environments. Such users can be better supported in making the right decisions when using nudges. Such users might be more sensitive in terms of nudge preferences. Users that do not care about privacy aspects might not be interested in customizing privacy nudges to their preferences. However, this group of users might also be more sensitive about their privacy data when default or presentation \& framing are used. Especially 
colored nudges might be interesting for this group of users because they do not really have to think about changes they have to make to better care about their privacy data. In order to avoid negative consequences, privacy nudges, for example, could only occur in certain situations that the individual has defined in their settings [3]. For users that are interested in privacy data, it is advantageous to leave the configuration of the privacy nudges, since they can adapt them to their individual needs and, above all, to their work situation. For example, the countdown for the time delay in the settings could be extended or shortened. In this area, research experiments have also shown that configuration options for privacy nudges are desired. This primarily concerns the timing of the nudges, which can have a strong influence on their perception [48, 47, 28]. Timing is also a relevant topic in the context of work, since the protection of privacy is often only of secondary priority and time must be devoted, above all, to work activities. Thus, it is important to get a better understanding about privacy nudges in digital environment and at the same time to analyze which users are interested in privacy issues.

In summary, our research provides several theoretical and practical contributions. First, we contribute to nudging literature by presenting an adapted classification of privacy nudges that is relevant for online environments. In addition, we contribute to theory by analyzing user preferences towards different kinds of nudges. With our ranking, researchers are now able to analyze each nudge in more detail to get a better understanding about their psychological relevance.

We support practitioners in developing nudging concepts based on the preferences of users. Thus, we deliver suggestions about which nudges to use when creating a privacy concept for online environments. Furthermore, with presenting the method of BWS, we present new opportunities in getting a better understanding about users and their preferences towards different objects. BWS is not limited to analyze digital nudges. It also allows further analyses of different objects in online environments. Thus, we support practitioners in conducting a BWS for their own purposes and analyses of objects in digital environments.

\section{Limitations and Future Research}

Lastly, we address the respective limitations of our work and objectives for future research. In our typology of privacy nudges, we distinguish between seven types of privacy nudges, but research has shown that individual nudges within a group have different goals and are considered to be context dependent. For example, colored presentation \& framing elements are used in a variety of designs and the privacy nudge of information covers a variety of designs to support privacy protection. Being able to take these differences into account, the extension of the typology into a taxonomy may be worth future research. In this context, a taxonomy could also take the related concept of gamification into account and how game mechanics relate to digital nudges in the privacy context [49].

Further limitations concern the BWS. BWS is a suitable method for analyzing user preferences [40], but the significance of the results depends, among other things, on the composition of the participant pool. In the present study, it was largely made up of students $(67.79 \%)$, while the proportion of full-time employees was comparatively small (17.51\%). The average age of the respondents was also rather low at 26.85 years. In this respect, a repeated survey could address full-time employees of all age groups to confirm the general validity of the results. In addition, we only used the BWS to determine user preferences for privacy nudges, but we did not determine their effectiveness in digital work environments. For example, a proposition whether the best rated default nudge is also the most effective privacy nudge cannot be stated.

Furthermore, digital work environments are used by organizations for many different functions and purposes. Thus, the restriction of the survey to the business messenger Slack can also represent a limitation. In addition, by using a BWS we provide room for future analyses by combining them with an experiment. These two aspects can be addressed in the future, ideally by testing individual privacy nudges in different digital work environments with field or online experiments. In addition, future research should focus on analyzing how the designs of individual nudges affect the user's reaction and privacy-related decisions. Especially the nudges that are not preferred by users should be analyzed in more detail to understand how to design them in a more attractive and meaningful way for users and to understand which designs of nudges might not be useful for a specific context such as the context of privacy. Finally, we could not analyze the psychological effects of privacy nudges in relation to users' preferences. We can just assume which kind of psychological aspects matter when using specific nudges. Therefore, future research could conduct experiments by analyzing which psychological effects are aroused in users when using a specific nudge. Such experiments could refer to the most preferred as well as the least preferred nudges to compare the users' reactions. In addition, regarding the usage of a BWS, future research could examine if ranking positions differ when other privacy aspects are considered or when other contexts are of relevance. Releasing data via social media or in sales might also be interesting to better 
understand the role of nudges in digital environments in relation to user preferences.

\section{Conclusion}

The goal of our study was to understand the role and meaning of user preferences towards different designs of privacy nudges in online environments. The results of our study indicate that users prefer nudges that are based on a visual design and colors such as red and green elements. Nudges that are based on textual elements, like information or feedback, are not preferred by users. These nudges might be more challenging to a user's working memory. Presentation \& framing nudges such as colors are easier to understand and help users in making faster decisions about publishing privacy-related data. Finally, elements that are based on pressure such as time delay should be used carefully when designing an IS because users do not prefer such nudges. They might be difficult to use in terms of privacy-related decisions.

\section{Acknowledgements}

The research presented in this paper was funded by the German Federal Ministry of Education and Research in the context of the project Nudger (www.nudger.de), Grant No. 16KIS0890K.

\section{References}

[1] Mirsch, T., C. Lehrer, and R. Jung, "Digital nudging: Altering user behavior in digital environments", Proceedings der 13. Internationalen Tagung Wirtschaftsinformatik (WI 2017), 2017, pp. 634-648.

[2] Harbach, M., M. Hettig, S. Weber, and M. Smith, eds., Using personal examples to improve risk communication for security \& privacy decisions, ACM, 2014.

[3] Acquisti, A., I. Adjerid, R. Balebako, L. Brandimarte, L.F. Cranor, S. Komanduri, P.G. Leon, N. Sadeh, F. Schaub, and M. Sleeper, "Nudges for privacy and security: Understanding and assisting users' choices online", ACM Computing Surveys (CSUR), 50(3), 2017, pp. 1-41.

[4] Lembcke, T.-B., N. Engelbrecht, A.B. Brendel, B. Herrenkind, and L.M. Kolbe, "Towards a Unified Understanding of Digital Nudging by Addressing its Analog Roots", Pacific Asia Conference on Information Systems (PACIS), 2019.

[5] Egelman, S. and E. Peer, eds., The myth of the average user: Improving privacy and security systems through individualization, ACM, 2015.

[6] Sunstein, C.R., "Nudging: A very short guide", Journal of Consumer Policy, 37(4), 2014, pp. 583-588.
[7] Kaiser, D., Individualized Choices and Digital Nudging: Multiple Studies in Digital Retail Channels, 2018.

[8] Louviere, J.J., I. Lings, T. Islam, S. Gudergan, and T. Flynn, "An introduction to the application of (case 1) bestworst scaling in marketing research", International Journal of Research in Marketing, 30, 2013, pp. 292-303.

[9] Thaler, R.H. and C.R. Sunstein, Nudge: Improving decisions about health, wealth, and happiness, Penguin, 2009.

[10] Hummel, D. and A. Maedche, "How effective is nudging? A quantitative review on the effect sizes and limits of empirical nudging studies", Journal of Behavioral and Experimental Economics, 80, 2019, pp. 47-58.

[11] Kroll, T. and S. Stieglitz, "Digital nudging and privacy: improving decisions about self-disclosure in social networks", Behaviour \& Information Technology, 2019, pp. 1-19.

[12] Weinmann, M., C. Schneider, and J. Vom Brocke, "Digital nudging", Business \& Information Systems Engineering, 58(6), 2016, pp. 433-436.

[13] Meske, C. and T. Potthoff, "The DINU-model-a process model for the design of nudges", European Conference on Information Systems (ECIS), 2017.

[14] Kissmer, T., T. Potthoff, and S. Stieglitz, "Enterprise Digital Nudging: Between adoption gain and unintended rejection", Americas Conference on Information Systems (AMCIS), 2018.

[15] Fogg, B.J., "Persuasive technology: using computers to change what we think and do", Ubiquity, 2002, pp. 89 120.

[16] Mirsch, T., C. Lehrer, and R. Jung, "Making Digital Nudging Applicable: The Digital Nudge Design Method", 2018.

[17] Wisniewski, P.J., B.P. Knijnenburg, and H.R. Lipford, "Making privacy personal: Profiling social network users to inform privacy education and nudging", International Journal of human-computer studies, 98, 2017, pp. 95-108.

[18] Krasnova, H., S. Spiekermann, K. Koroleva, and T. Hildebrand, "Online social networks: Why we disclose", Journal of Information Technology, 25(2), 2010, pp. 109125.

[19] Hui, K.-L., B.C.Y. Tan, and C.-Y. Goh, "Online information disclosure: Motivators and measurements", ACM Transactions on Internet Technology (TOIT), 6(4), 2006, pp. 415-441.

[20] Caraban, A., E. Karapanos, D. Gonçalves, and P. Campos, "23 Ways to Nudge: A Review of TechnologyMediated Nudging in Human-Computer Interaction", CHI Conference on Human Factors in Computing Systems Proceedings, 2019.

[21] Kahneman, D., "Thinking, fast and slow (1st pbk. ed.)", New York: Farrar, Straus and Giroux, 2013.

[22] Hertwig, R. and T. Grüne-Yanoff, "Nudging and boosting: Steering or empowering good decisions", 
Perspectives on Psychological Science, 12(6), 2017, pp. 973-986.

[23] Tversky, A. and D. Kahneman, "Judgment under uncertainty: Heuristics and biases", science, 185(4157), 1974, pp. 1124-1131.

[24] Sojka, J.Z. and J.L. Giese, "Communicating through pictures and words: Understanding the role of affect and cognition in processing visual and verbal information", Psychology \& Marketing, 23(12), 2006, pp. 995-1014.

[25] Vom Brocke, J., A. Simons, K. Riemer, B. Niehaves, R. Plattfaut, and A. Cleven, "Standing on the Shoulders of Giants: Challenges and Recommendations of Literature Search in Information Systems Research.", Communications of the Association for Information Systems, 37(9), 2015, pp. 205-244.

[26] Turland, J., L. Coventry, D. Jeske, P. Briggs, and A. van Moorsel, eds., Nudging towards security: Developing an application for wireless network selection for android phones, ACM, 2015.

[27] Wang, Y., P.G. Leon, A. Acquisti, L.F. Cranor, A. Forget, and N. Sadeh, eds., A field trial of privacy nudges for facebook, ACM, 2014.

[28] Liu, B., M.S. Andersen, F. Schaub, H. Almuhimedi, S.A. Zhang, N. Sadeh, Y. Agarwal, and A. Acquisti, eds., Follow my recommendations: A personalized privacy assistant for mobile app permissions, 2016.

[29] Khern-am-nuai, W., W. Yang, and N. Li, "Using Context-Based Password Strength Meter to Nudge Users' Password Generating Behavior: A Randomized Experiment", 2017.

[30] Chang, D., E.L. Krupka, E. Adar, and A. Acquisti, eds., Engineering information disclosure: Norm shaping designs, ACM, 2016.

[31] Coventry, L.M., D. Jeske, J.M. Blythe, J. Turland, and P. Briggs, "Personality and social framing in privacy decision-making: A study on cookie acceptance", Frontiers in psychology, 7, 2016, p. 1341.

[32] Bakshy, E., D. Eckles, R. Yan, and I. Rosenn, eds., Social influence in social advertising: evidence from field experiments, ACM, 2012.

[33] Flynn, T.N., J.J. Louviere, T.J. Peters, and J. Coast, "Best-worst scaling: What it can do for health care research and how to do it", Journal of Health Economics, 26, 2007, pp. 171-189.

[34] Thurstone, L.L., "A Law of Comparative Judgement", Psychological Review(4), 1927, pp. 273-286.

[35] Finn, A. and J.J. Louviere, "Determining the Appropriate Response to Evidence of Public Concern: The Case of Food Safety", Journal of Public Policy \& Marketing, 11(1), 1992, pp. 12-25.

[36] Marley, A.A.J. and J.J. Louviere, "Some probabilistic models of best, worst, and best-worst choices", Journal of Mathematical Psychology, 49(6), 2005, pp. 464-480.
[37] Lee, J.A., G.N. Soutar, and J. Louviere, "Measuring values using best-worst scaling: The LOV example", Psychology \& Marketing, 24(12), 2007, pp. 1043-1058.

[38] Matzner, M., M. von Hoffen, T. Heide, F. Plenter, and F. Chasin, "A Method for Measuring User Preferences in Information Systems Design Choices", Twenty-Third European Conference on Information Systems (ECIS), 2015.

[39] Lu, X., R.B. Adams, J. Li, M.G. Newman, and J.Z. Wang, eds., An investigation into three visual characteristics of complex scenes that evoke human emotion, IEEE, 2017.

[40] Cohen, S.H., "Maximum Difference Scaling: Improved Measures of Importance and Preferences for Segmentation", Sawtooth Research Paper Series, 2003, pp. 117.

[41] Lansing, J., S. Schneider, and A. Sunyaev, "Cloud Service Certification: Measuring Consumers' Preferences For Assurances", European Conference on Information Systems, 2013.

[42] Severin, F., J. Schmidtke, A. Mühlbacher, and W.H. Rogowski, "Eliciting preferences for priority setting in genetic testing: a pilot study comparing best-worst scaling and discrete-choice experiment", European Journal of Human Genetics, 21, 2013, pp. 1202-1208.

[43] Orme, B., "Accuracy of HB Estimation in MaxDiff Experiments", Sawtooth Research Paper Series, 2005, pp. 1-7.

[44] Oppenheimer, D.M., T. Meyvis, and N. Davidenko, "Instructional manipulation checks: Detecting satisficing to increase statistical power", Journal of experimental social psychology, 45(4), 2009, pp. 867-872.

[45] Hu, P.J.-H., H.-f. Hu, and X. Fang, "Examining the Mediating Role of Cognitive Load and Performance Outcomes in User Satisfaction with a Website: A Field QuasiExperiment", MIS-Quarterly, 41(3), 2017, pp. 975-987.

[46] Knijnenburg, B. and H. Jin, "The persuasive effect of privacy recommendations for location sharing services", Available at SSRN 2399725, 2013.

[47] Micallef, N. and N.A.G. Arachchilage, "A Serious Game Design: Nudging Users' Memorability of Security Questions", arXiv preprint arXiv:1709.08167, 2017.

[48] Almuhimedi, H., F. Schaub, N. Sadeh, I. Adjerid, A. Acquisti, J. Gluck, L.F. Cranor, and Y. Agarwal, eds., Your location has been shared 5,398 times!: A field study on mobile app privacy nudging, ACM, 2015.

[49] Schöbel, S. and A. Janson, "Is it all about Having Fun? - Developing a Taxonomy to Gamify Information Systems", European Conference on Information Systems (ECIS), 2018. 